\title{
What's new for the clinician? - Excerpts from and summaries of recently published papers
}

\section{The effects of a hydrogen peroxide mouthrinse on the intraoral viral load of SARS-CoV-2}

MJ Gottsauner, I Michaelides, B Schmidt, et al. A prospective clinical pilot study on the effects of a hydrogen peroxide mouthrinse on the intraoral viral load of SARS-CoV-2. Clinical oral investigations. 2020; 2 Sep: 1-7.

\section{INTRODUCTION}

The most common biological complication around dental implants is peri-implant mucositis, which can be found in about $64.6 \%$ of all implant patients. ${ }^{1}$ Peri-implant mucositis is defined as an inflammation of the periimplant soft tissues with Bleeding on Probing (BOP), without loss of supporting bone, with or without increased Probing Depth (PD) and with visual signs of inflammation. ${ }^{1}$

Untreated peri-implant mucositis can progress to periimplantitis with progressive soft tissue and bone loss. The use of homecare measures such as brushing, mouthrinsing, the use of oral irrigators, etc. have all been used to maintain good oral hygiene and reduce plaque load. Bunk and colleagues (2020) ${ }^{1}$ reported on a trial that sought to determine whether the adjuvant use of an oral irrigation device with chlorhexidine $(\mathrm{CHX})$ as part of self-administered plaque control significantly reduces severity and presence of peri-implant mucositis compared to no irrigation or irrigation with water.

\section{MATERIALS AND METHODS}

The was a prospective clinical study investigating the effects of a mouthrinse with $1 \%$ hydrogen peroxide on the intraoral viral load of hospitalized SARS-CoV-2-positive patients in an isolation ward.

Only patients with a positive test for SARS-CoV-2 within the last $72 \mathrm{~h}$ were included in this study. Exclusion criteria were indication for intubation or mechanical ventilation and severe stomatitis.

Veerasamy Yengopal: $B C h D, B S c H o n s, M C h D, P h D$, Community Dentistry Department, School of Oral Health Sciences, University of Witwatersrand, Medical School, no. 7 York Road, Parktown 2193, South Africa.

ORCID Number: 0000-0003-4284-3367

Email: veerasamy.yengopal@wits.ac.za
Patients were screened for eligibility by one medical doctor and provided with detailed description of the study outline which involved the following procedure: patients were asked to gargle their mouth and throat with $20 \mathrm{~mL} 0.9 \% \mathrm{NaCl}$ for $30 \mathrm{~s}$ for acquiring a baseline oropharyngeal specimen for the SARS-CoV-2 realtime PCR (RT-PCR) test. Immediately afterwards, patients had to perform a mouthrinse with $20 \mathrm{~mL} 1 \%$ hydrogen peroxide by gargling their mouth and throat for 30 s. Thirty minutes after this mouthrinse, another oropharyngeal specimen for the SARS-CoV-2 RT-PCR test was acquired by letting the patients gargle their mouth and throat with $20 \mathrm{~mL} 0.9 \% \mathrm{NaCl}$ for $30 \mathrm{~s}$. The respective quantities of copies/mL of SARS-CoV-2 RNA were analyzed by RT-PCR.

SARS-CoV-2 was isolated from the oropharyngeal specimens that exhibited more than 103 copies/mL of SARSCoV-2 RNA at baseline by using kidney epithelial cells from African green monkey (Vero-CCL19 cells, ATCC). Data are reported as median values (with $1^{\text {st }}$ and $3^{\text {rd }}$ quartiles) and were statistically analyzed non-parametrically using the Wilcoxon signed-rank test for related samples on a significance level of $\alpha=0.05$.

\section{RESULTS}

Twelve SARS-CoV-2-positive patients were included in this study. These 12 patients ( 6 female and 6 male) had a median age of 55 years (range: $22-81$ years). One patient was hospitalized in an intensive care unit (without need of intubation), and 11 were hospitalized in an isolation ward. Eleven out of the 12 patients showed comorbidities (e.g., diseases of the liver, cardiovascular system or kidney, haematological diseases, and obesity).

In two out of the 12 initially included patients, no SARSCoV-2 RNA could be detected in the baseline specimens prior to performing the $1 \%$ hydrogen peroxide mouth- 
rinse. Therefore, these two patients were excluded from the study. There were no significant differences between baseline viral load and viral load $30 \mathrm{~min}$ after the 1\% hydrogen peroxide mouthrinse $(p=0.96)$.

\section{CONCLUSIONS}

The researchers concluded that a $1 \%$ hydrogen peroxide mouthrinse does not reduce the intraoral viral load in SARS-CoV-2-positive subjects.

\section{Implications of practice}

The recommendation of a preprocedural mouthrinse with hydrogen peroxide before intraoral procedures is questionable and thus should not be supported any longer, but strict infection prevention regimens remain crucial for patient safety.

\section{Reference}

1. Gottsauner MJ, Michaelides I, Schmidt B, et al. A prospective clinical pilot study on the effects of a hydrogen peroxide mouthrinse on the intraoral viral load of SARS-CoV-2. Clinical oral investigations. 2020; Sep 2: 1-7.

\section{The effect of adjuvant oral irrigation on self-administered oral care in the management of peri-implant mucositis.}

D Bunk, M Eisenburger, S Häckl, et al. The effect of adjuvant oral irrigation on self-administered oral care in the management of peri-implant mucositis: A randomized controlled clinical trial. Clinical Oral Implants Research. 2020 Oct.

\section{INTRODUCTION}

The most common biological complication around dental implants is peri-implant mucositis, which can be found in about $64.6 \%$ of all implant patients. ${ }^{1}$ Peri-implant mucositis is defined as an inflammation of the periimplant soft tissues with Bleeding on Probing (BOP), without loss of supporting bone, with or without increased Probing Depth (PD) and with visual signs of inflammation. ${ }^{1}$ Untreated peri-implant mucositis can progress to peri-implantitis with progressive soft tissue and bone loss.

The use of homecare measures such as brushing, mouthrinsing, the use of oral irrigators, etc. have all been used to maintain good oral hygiene and reduce plaque load. Bunk and colleagues (2020) ${ }^{1}$ reported on a trial that sought to determine whether the adjuvant use of an oral irrigation device with chlorhexidine $(\mathrm{CHX})$ as part of self-administered plaque control significantly reduces severity and presence of peri-implant mucositis compared to no irrigation or irrigation with water.

\section{MATERIALS AND METHODS}

This was a randomized controlled clinical trial that consisted of adult participants suffering from peri-implant mucositis who were recruited during regular oral maintenance and treatment appointments at an implant dental clinic. Study participants met the following inclusion criteria: (a) presence of at least one dental implant in function for $>12$ months; (b) clinical diagnosis of peri-implant mucositis at one or more implant sites with (bleeding on probing) BOP with or without suppuration; (c) absence of radiographic bone loss when compared to previous radiographs; (d) visual signs of inflammation $(m G l \neq 0)$ at one or more implant sites.

Exclusion criteria were: (a) Peri-implantitis, which was either diagnosed with progressive bone loss comparing previous and current radiographs, or in case of missing previous data or radiographs, with bone levels $\geq 3 \mathrm{~mm}$ apical of the most coronal portion of the intraosseous part of the implant; (b) smoking or chewing tobacco, alcohol- or drug addiction; (c) systemic diseases or conditions such as metabolic bone- and haematological diseases, immunodeficiency, uncontrolled diabetes mellitus, kidney failure, risk of endocarditis, tumour or radiation in head and neck area; (d) systemic antibiotics use 3 months prior to baseline, steroid and coumarin therapy 3 months prior to baseline; and (e) physical limitations that prevent adequate oral hygiene technique.

At baseline, clinical measurements were carried out and all subjects were given a standardized basic oral hygiene instruction in verbal and written form. The basic oral hygiene instructions contained a description of the recommended brushing technique and cleaning of removable dentures where applicable. After this sequence, the patients were instructed to brush again with a small amount of fluoride-containing toothpaste. The written oral hygiene instruction additionally contained instructions for interproximal cleaning. The patients were given additional information about the consequences of progression of peri-implant mucositis and transition into peri-implantitis. A sub- and supramucosal mechanical debridement with titanium curettes and polishing using a rubber cup and low abrasive polishing paste were performed at baseline.

Patients assigned to group 1 did not receive any additional instruction or demonstration and were advised to follow the basic oral hygiene instructions independently at home. Patients assigned to group 2 and 3 were given a demonstration and written instructions for the use of an oral irrigation system (Waterpik $($ ). Patients of group 2 received bottles with in total $4.5 \mathrm{~L}$ of water and patients 
of group 3 received bottles with in total 4.5 litres of $0.06 \%$ Chlorhexidine solution. Patients of group 2 and 3 were instructed to use the oral irrigator $1 \mathrm{x} /$ day with $50 \mathrm{ml}$ of the respective solution after toothbrushing and interproximal cleaning in the evenings at the implant. Toothbrushes and fluoride-containing toothpaste were handed out to all subjects at baseline visit.

All patients returned for follow-up examination and data collection after 4,8 , and 12 weeks. The follow-up visits included measurement of clinical data and a standardized protocol for reinstruction and remotivation. Patients in group 2 and 3 also received additional quantities of the assigned irrigation solution.

Only one implant per individual was included in the study. If more than one implant per patient was diagnosed with peri-implant mucositis, the implant with the highest mucositis severity score was chosen for the study. Clinical data were measured at four sites around the implant (mesio-buccal, disto-buccal, mesio-lingual, disto-lingual).

The following clinical parameters were assessed: (a) PD in $\mathrm{mm}$ with a standard probe; (b) Bleeding on Probing (BOP); (c) Modified Gingival Index for dental implants (mGl); (d) Modified Plaque Index for dental implants (mPI); (e) mucositis severity score. The mucositis severity score reaches from 0-16. It is calculated based on the sum of the mGl-score from 4 implant sites (maximum 12 points - as the $\mathrm{mGl}$ reaches from $0-3$ ) and the BOPpositive sites (maximum 4 points, if $\mathrm{BOP}$ is present on all 4 examined implant sites. All parameters were measured in each patient at each appointment.

\section{RESULTS}

A total of 72 patients were assessed for eligibility for inclusion into this trial. Twelve patients did not meet the inclusion criteria and were excluded: six patients were smokers, four patients suffered from peri-implantitis, two patients had a risk of endocarditis. At baseline examination, no significant differences among the groups were observed for age, sex, implant region, dentures, mucositis severity score, and PDs.

At the 12-weeks follow-up, the prevalence of periimplant mucositis of all examined implants was $30 \%$. The lowest prevalence of peri-implant mucositis was found in group 3 with 5\%, followed by group 2 (35\%) and group 1 (50\%).

Patients of group 3 showed significantly lower BOPpositive sites after 12 weeks when compared to control group $(-0.75$ [95\% Cl -1.26; -0.25], $p=.004)$. No significantly lower BOP-positive sites could be found when oral irrigation with $\mathrm{CHX}$ was compared to water $(-0.36$ [95\% Cl -0.86; 0.14], $p=.16)$, or when water irrigation was compared to control group $(-0.39$ [95\% $\mathrm{Cl}-0.9 ; 0.11], p=.12)$.

Groups 2 and 3 showed a considerably higher reduction of mucositis severity score after 12 weeks compared to control group. The final examination showed a mean mucositis severity score of mild inflammation for groups 2 and 3 and moderate inflammation in group 1.
The severity of peri-implant mucositis, based on the mucositis severity score after 12 weeks, varied significantly between the groups. The linear regression analysis showed a significant improvement of the severity of periimplant mucositis when using $\mathrm{CHX}$ irrigation compared to oral hygiene instructions only $(-2.4[95 \% \mathrm{Cl}-4.19$; $-0.61], p=.001)$. The use of an irrigation device with water compared to control resulted in an estimated drop of 1.7 points in mucositis severity score after 12 weeks closely to the significance threshold $(-1.7$ [95\% $\mathrm{Cl}-3.49 ; 0.1], p=.06)$, however, but was not significant.

The highest mean decrease of average $\mathrm{mPI}$ from baseline to final examination was observed in group 1; however, the scores of group 2 and 3 were very close to that. The results of the linear regression for $\mathrm{mPI}$ showed no significant influence of the three interventions $(p=.99)$ on the outcome. Except for $\mathrm{mPI}$, each regression model showed superiority of the use of an oral irrigator with $\mathrm{CHX}$ compared to control group.

The hypothesis, that the adjuvant use of $\mathrm{CHX}$ in an oral irrigation device significantly reduces clinical signs of peri-implant mucositis compared to no oral irrigation or water irrigation was confirmed.

\section{CONCLUSIONS}

The researchers concluded that the adjuvant use of an oral irrigator with $0.06 \% \mathrm{CHX}$ in addition to mechanical biofilm removal can reduce severity and presence of peri-implant mucositis after 12 weeks.

\section{Implications for practice}

The use of $\mathrm{CHX}$ instead of water as oral irrigator solution seems to offer significantly better clinical outcomes in patients with peri-implant mucositis. However, the possible long-term effect of $\mathrm{CHX}$ use must be noted by clinicians.

\section{Reference}

1. Bunk D, Eisenburger $M$, Häckl S, Eberhard J, Stiesch $M$, Grischke J. The effect of adjuvant oral irrigation on selfadministered oral care in the management of peri-implant mucositis: A randomized controlled clinical trial. Clinical Oral Implants Research. 2020 Oct. 